\title{
Interacting coronae of two T Tauri stars: first observational evidence for solar-like helmet streamers
}

\author{
M. Massi ${ }^{1}$, E. Ros ${ }^{1}$, K. M. Menten ${ }^{1}$, M. Kaufman Bernadó ${ }^{1, \star}$, G. Torricelli-Ciamponi ${ }^{2}$, \\ J. Neidhöfer ${ }^{1}$, A. Boden ${ }^{3}$, D. Boboltz ${ }^{4}$, A. Sargent ${ }^{5}$, and G. Torres ${ }^{6}$ \\ 1 Max-Planck-Institut für Radioastronomie, Auf dem Hügel 69, 53121 Bonn, Germany \\ e-mail: [mmassi;ros;kmenten;mkaufman; jneidhoefer] @mpifr-bonn.mpg.de \\ 2 INAF - Osservatorio Astrofisico di Arcetri, Largo E. Fermi 5, 50125 Firenze, Italy \\ e-mail: torricel@arcetri.astro.it \\ 3 Michelson Science Center, California Institute of Technology, 770 South Wilson Ave., Pasadena CA 91125, USA \\ e-mail: bode@ipac.caltech.edu \\ 4 US Naval Observatory, 3450 Massachusetts Ave, Washington DC 20392, USA \\ e-mail: dboboltz@usno.navy.mil \\ 5 Division of Physics, Math, and Astronomy, California Institute of Technology, MS 105-24, Pasadena, CA 91125, USA \\ e-mail: afs@astro.caltech.edu \\ ${ }^{6}$ Harvard-Smithsonian Center for Astrophysics, 60 Garden St., Cambridge, MA 02138, USA \\ e-mail: gtorres@cfa.harvard.edu
}

Received 7 September 2007 / Accepted 13 November 2007

\begin{abstract}
Context. The young binary system V773 Tau A exhibits a persistent radio flaring activity that gradually increases from a level of a few $\mathrm{mJy}$ at apoastron to more than $100 \mathrm{mJy}$ at periastron. Interbinary collisions between very large $\left(>15 R_{*}\right)$ magnetic structures anchored on the two rotating stars of the system have been proposed to be the origin of these periodic radio flares. Magnetic structures extended over tens of stellar radii, that can also account for the observed fast decay of the radio flares, seem to correspond to the typical solar semi-open quite extended magnetic configurations called helmet streamers.

Aims. We aim to find direct observational evidence for the postulated, solar-like, coronal topologies.

Methods. We performed seven-consecutive-day VLBI observations at $8.4 \mathrm{GHz}$ using an array consisting of the VLBA and the 100-m Effelsberg telescope. V773 Tau A was phase-referenced to QSO B0400+258.

Results. Two distinctive structures appear in the radio images here presented. They happen to be associated with the primary and secondary stars of the V773 Tau A system. In one image (Fig. 2B) the two features are extended up to $18 R_{*}$ each and are nearly parallel revealing the presence of two interacting helmet streamers. One image (Fig. 2E) taken a few hours after a flare monitored by the 100-m Effelsberg telescope shows one elongated fading structure substantially rotated with respect to those seen in the B run. The same decay scenario is seen in Fig. $2 \mathrm{G}$ for the helmet streamer associated with the other star.

Conclusions. This is the very first direct evidence revealing that even if the flare origin is magnetic reconnection due to interbinary collision, both stars independently emit in the radio range with structures of their own. These structures are helmet streamers, observed for the first time in stars other than the Sun. The complete extent of each helmet streamer above the stellar surface is about $24 R_{*}$ which implies that they can practically interact throughout the whole orbit, even rather close to apoastron where the stellar separation is $52 R_{*}$. However, the radio flares become stronger when the stars approach. Around periastron the stellar separation is only $30 R_{*}$, nearly covered by a single streamer: the two streamers overlap producing the observed giant flares.
\end{abstract}

Key words. stars: coronae - stars: individual: V773 Tau - stars: flare - stars: pre-main sequence - radio continuum: stars stars: binaries: general

\section{Introduction}

Located in the Taurus-Auriga star forming region, at a distance of $136 \mathrm{pc}$, the young binary system V773 Tau A (see Fig. 1) is formed by two stars of $1.5 M_{\odot}$ and $1.3 M_{\odot}$ (Boden et al. 2007). This system is a highly variable radio source: it exhibits persistent flaring activity that gradually increases from its weakest level at apoastron, few mJy, to its strongest at periastron, more than $100 \mathrm{mJy}$. The strong correlation observed between the flare intensity and the distance between the two stars suggests the occurrence of interbinary collisions of the binary components' very extended coronae (Massi et al. 2002, 2006). Knowing that the distance between the two stars at apoastron and at periastron is

^ Humboldt Research Fellow.
$52 R_{*}$ and $30 R_{*}$, respectively $\left(R_{*}\right.$ stellar radius, $R_{*} \simeq 2 R_{\odot}$ for both stars, which corresponds to 0.069 mas at $136 \mathrm{pc}$, Boden et al. 2007), interbinary collisions would then imply very large coronal structures with a size of $15 R_{*} \leq H \leq 26 R_{*}$. On the other hand, X-ray observations have provided clear evidence for much smaller coronal loops with sizes of 0.6-1.4 $R_{*}$ (Skinner et al. 1997; Tsuboi et al. 1998). Although the radio and X-ray observations might appear contradictory, a multiwavelength campaign carried out on this system (Feigelson et al. 1994) proved that the radio and X-ray emissions come from spatially separated regions. In fact this study showed that during radio variability the $\mathrm{X}$-ray flux remained steady.

This implies that the coronae in the V773 Tau A stellar system are formed by at least two different types of magnetic 


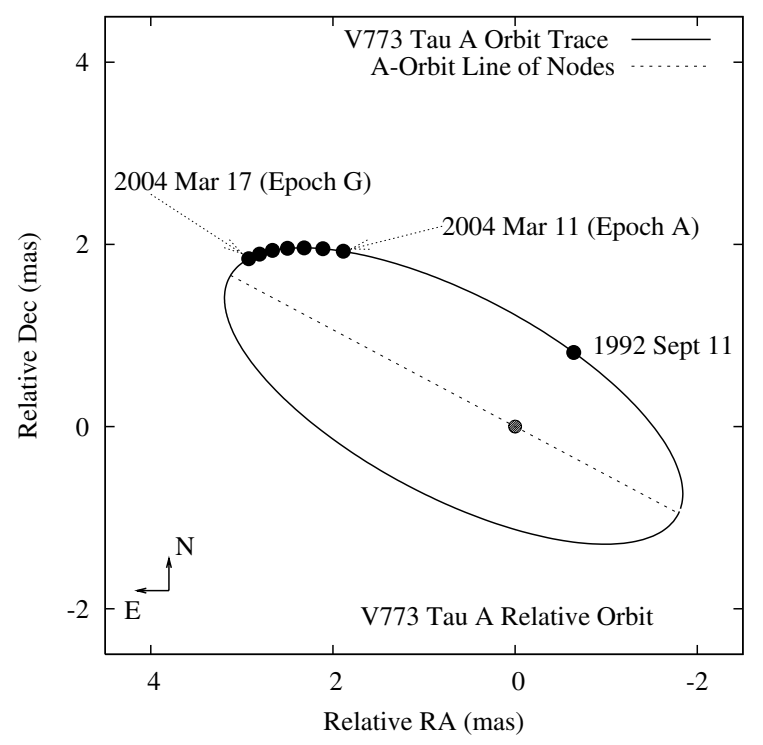

Fig. 1. Orbit of the young stellar binary system V773 Tau based on the interferometric-spectroscopic model by Boden \& collaborators (2007). The secondary is given at our seven (A-G) VLBI observations (epoch 2004) and at Phillips \& collaborators (1996) VLBI observation (epoch 1992). Stellar diameters are rendered to scale.

structures: one, stellar sized, associated with the X-ray emission and another, extended, associated with the radio emission. In the very well studied solar corona it is known that there exist small, closed coronal loops as well as much more extended semi-open structures called helmet streamers. Solar helmet streamers are formed by a helmet, i.e. a cusp-shaped field line located on top ( 2 to $4 R_{\odot}$ ) of a coronal loop, and a very long streamer which extends out to $30 R_{\odot}$ as can be seen in optical images from the Solar and Heliospheric Observatory (SOHO) satellite (Schwenn 2006; Suess \& Nerney 2004; Vourlidas 2006). Following the analogy with the Sun, Massi \& collaborators (2006) proposed a model where helmet streamers are the basic magnetic topology at the origin of the observed flares of the V773 Tau A system. This model with a helmet located at $2-5 R_{*}$ and an upper mirror point at the top of a streamer of $10-20 R_{*}$, was able to explain the fading phase of the flares in V773 Tau A in the centimeter and millimeter radio band. In this scenario the extended streamers collide and magnetic reconnection takes place far out from the stellar surfaces. Therefore, the radio flares in V773 Tau A depend on magnetic interactions like in the case of the solar flares, with the difference that the interacting magnetic structures belong to the two distinct coronae of each of the stars of the binary system. These interacting magnetic structures are streamers, most likely located at the top of the observed stellar sized X-ray emitting coronal loops.

At this point, it is important to mention the large radio emitting structure imaged during a Very-Long-Baseline Interferometry (VLBI) observation carried out close to the periastron passage of V773 Tau A in 1992 by Phillips \& collaborators (1996, see Fig. 1). This VLBI image shows a large halo ( $\geq 3$ mas) surrounding two compact components that are separated by 1.14 mas. The two components have been interpreted by Phillips \& collaborators as emitting zones associated with each of the two stars of the binary system. In fact, the separation of 1.14 mas, i.e. $16 R_{*}$, inferred from their observation is completely consistent with the projected stellar separation of 1.03 mas, i.e. $15 R_{*}$ (see Fig. 1). How is this halo related to the postulated helmet streamers? Are the streamers existing as stable independent magnetic structures or are they continuously merged together forming the observed halo?

In order to investigate this complex coronal topology we decided to perform a long (seven-consecutive-day) set of new VLBI observations at a time when the system is quite displaced from periastron, so that the larger distances between the two stars could reduce the overlap between the streamers and therefore the formation of joint structures. We describe these new VLBI observations and our results in Sects. 2 and 3. In Sect. 3.1 we discuss the position of the two stars of the binary system with respect to the radio peaks present in each observation. In Sect. 3.2 we interpret the resolved structures as helmet streamers, in Sect. 3.3 we discuss the possible distribution of helmet streamers around the stellar surface, and in Sect. 3.4 we focus on the short-living upper mirror points closing the streamers. In Sect. 4.1 the different flaring activity at apoastron and periastron, and the different observed topologies are discussed on the basis of the here presented results. Finally, in Sect. 4.2, we discuss the importance of the helmet streamer configuration in Astrophysics.

\section{Observations and data reduction}

We observed V773 Tau A at $8.4 \mathrm{GHz}$ with an array consisting of the Very Long Baseline Array (VLBA, 10 telescopes of $25 \mathrm{~m}$ each across the USA) together with the Effelsberg 100-m radio telescope (VLBA+EB). The addition of Effelsberg provided long baselines and a higher sensitivity, together with the possibility of measuring the flux density of the target source in between the VLBI scans during the observation. The observing runs, listed as segments A to G (code BM198) in the first column of Table 1, covered the UT range from 17:30 to 23:00, on the JD time given in the second column. The 3rd column of the table gives the orbital phase calculated for the orbital period, $P=51.1033 \mathrm{~d}$, and the time periastron passage, $T_{0}=$ 53 059.50 MJD (Boden et al. 2007). The selected range of orbital phases, $\Phi=0.32-0.43$ implies larger distances between the two stars close to apoastron (see Fig. 1).

The data were recorded at each station over four $8-\mathrm{MHz}$ bands at a bit rate of $128 \mathrm{Mbps}$, in dual polarization with 1-bit sampling, yielding a total bandwidth of $32 \mathrm{MHz}$ for each polarization hand. The data were processed at the VLBA correlator at the NRAO's Array Operations Center in Socorro, New Mexico, using a pre-averaging time of $4 \mathrm{~s}$. We chose as reference source the QSO B0400+258 (CTD 26, J0403+2600), 3.3 ${ }^{\circ}$ apart from the target source V773 Tau A, and switched between both with cycles of $\sim 7 \mathrm{~min}$, observing $360 \mathrm{~s}$ at the target source and $90 \mathrm{~s}$ at the reference source. Before every fourth scan at the target source, Effelsberg measured the total flux density at the observing frequency.

We used a catalog position for $\mathrm{B} 0400+258$ of $\alpha_{\mathrm{J} 2000.0}=$ $04^{\mathrm{h}} 03^{\mathrm{m}} 05^{\mathrm{s}} .586056$ and $\delta_{\mathrm{J} 2000.0}=26^{\circ} 00^{\prime} 01^{\prime \prime} .50288$. The position used for V773 Tau was $\alpha_{\mathrm{J} 2000.0}=04^{\mathrm{h}} 14^{\mathrm{m}} 12^{\mathrm{s}} .919833$ and $\delta_{\mathrm{J} 2000.0}=28^{\circ} 12^{\prime} 12^{\prime \prime}{ }^{\prime} 19953$, after computing the proper motion from the reference epoch of 1993.88, with rates of $\Delta \alpha 0.42 \mathrm{mas} / \mathrm{yr}$ and $\Delta \delta-23.25 \mathrm{mas} / \mathrm{yr}$. We used the QSO 3C 84 as fringe finder, and additionally, to check the stability of the astrometry we also had blocks of observations of the reference source B0405+305 (J0408+3032). Polarization calibrators were DA 193 and B0059+581 (J0102+5824), and 4C 28.07 (CTD 20, $\mathrm{B} 0234+285$, J0237+2848).

The post-correlation data reduction was performed using the Astronomical Image Processing System ( $\mathcal{A} \mathcal{I P S})$. A priori visibility amplitude calibration was done using the antenna 


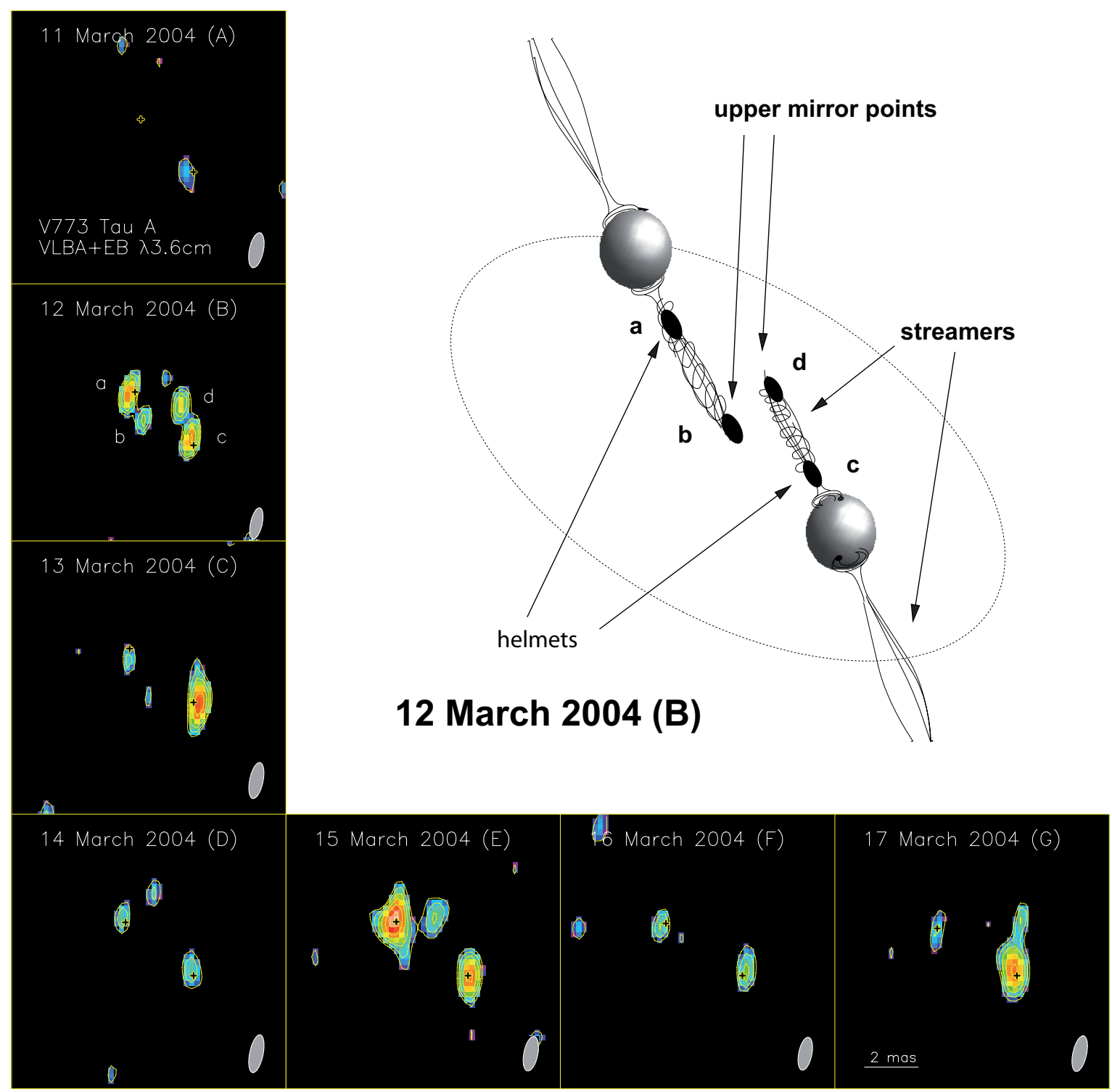

Fig. 2. Seven consecutive $8.4 \mathrm{GHz}$ VLBA+EB images of the young stellar binary system V773 Tau A. Stellar positions are indicated by crosses. Starting with panel A, the peak flux density in the images are $0.1,0.5,0.5,0.2,0.7,0.2,0.5 \mathrm{mJy} / \mathrm{beam}$. Contour levels are $-1,1,1.5,2,2.5,3,4$ and 5 times the $3 \sigma(0.1 \mathrm{mJy} /$ beam $)$. The beam size is shown in the bottom right corner of each panel (average beam size: $1.3 \times 0.5 \mathrm{mas})$. The sketch of the system, not to scale, shows helmet streamers, i.e. structures formed by a helmet located on top of a coronal loop anchored on a rotating star, and a very large streamer. The topology is here simplified, for each star are only drawn two streamers of a possible solar-like streamer belt.

gains and the system temperatures measured at each station. Instrumental phase offsets between the 8-MHz bands, caused by differing paths through the electronics, were determined using short section of data on the source DA 193 and applied to all of the data. To correct for any residual phase rates and delays, a fringe-fit was performed on the reference source, QSO B0400+258, and the resulting solutions were interpolated to the target source data. Finally, an iterative self-calibration and imaging procedure was performed on the reference source to correct for any residual phase and amplitude errors. The resulting corrections were then applied to the V773 Tau A data. The same calibration procedure was repeated for all the seven epochs of observations. With the calibration completed, $512 \times 512$ pixel total intensity (Stokes $I$ ) images of the target source were produced with a pixel spacing of 0.2 mas. The synthesized beam sizes for the 7 epochs were roughly the same at $\sim 1.4 \times 0.5$ mas. Figure 2 shows the images of V773 Tau A produced for all the seven epochs.

\section{Results and discussion}

\subsection{Radio peaks and position of the two stars}

Besides Fig. 2A, where only one weak $(3 \sigma)$ feature is present, two structures are always present in all the other images (Figs. 2B-G), here called North-East, NE, and South-West, SW. The two structures can be quite complex as in Fig. 2B where they show two components each ("a", "b" for the NE and "c", "d" for the SW). The integrated flux measured for the NE and SW features with the AIPS task JMFIT is given in Fig. 3 along with the total flux measured by the Effelsberg 100-m telescope. As can be seen in Fig. 3 the VLBI flux is very low, i.e. $\leq 1 \mathrm{mJy}$, and no significant fraction of circularly and linearly polarized radiation was detected. The higher level of total flux observed with the Effelsberg 100-m telescope, with a beam of $\sim 1.5$ arcmin, implies the existence of larger magnetic structures around those traced with VLBA+EB at milliarcsecond scales. The position of the brightest radio peak associated with each NE and SW feature 
Table 1. Stellar and helmet separations in the young stellar binary system V773 Tau A during each VLBA+EB observation. Julian day 2453076.34 corresponds to March 11, 2004 at 20:10. Ha and $\mathrm{Hb}$ are the two radio peaks in the VLBI images associated with the helmets. Aa and $\mathrm{Ab}$ are the primary (V773 Tau Aa) and secondary (V773 Tau Ab) stars of the system respectively. The error associated to the separations is \pm 0.1 mas. At a distance of $136 \mathrm{pc}$, one stellar radius, $R_{*}$, corresponds to 0.069 mas.

\begin{tabular}{ccccc}
\hline \hline Run & $\begin{array}{c}\text { Date } \\
\text { (JD) }\end{array}$ & $\Phi$ & $\begin{array}{c}\text { Ha-Hb } \\
\text { (mas) }\end{array}$ & $\begin{array}{c}\text { Aa-Ab } \\
\text { (mas) }\end{array}$ \\
\hline A & 2453076.34 & 0.32 & & 2.7 \\
B & 2453077.34 & 0.34 & 2.8 & 2.9 \\
C & 2453078.34 & 0.35 & 3.2 & 3.1 \\
D & 2453079.34 & 0.37 & 3.2 & 3.2 \\
E & 2453080.34 & 0.39 & 3.4 & 3.3 \\
F & 2453081.34 & 0.41 & 3.5 & 3.4 \\
G & 2453082.34 & 0.43 & 3.1 & 3.5 \\
\hline
\end{tabular}

were determined and their separations are given in the 4th column of Table 1.

The visual orbit derived from the interferometricspectroscopic orbital model of Boden et al. (2007) only shows (Fig. 1) the relative position between the two components of the system (i.e. their separation, see 5th column of Table 1), it does not automatically identify the position of both components on the radio maps.

Comparing the 4th and 5 th columns one can see that the separation between the two stars grows from 2.7 mas at run A to 3.5 mas at run $\mathrm{G}$. This is, in fact, consistent with the growing distance between the two radio peaks as well. Besides run $\mathrm{G}$, where there is a difference of 0.4 mas $\left(6 R_{*}\right)$ between the stellar separation and the separation of the radio peaks, all the other distances agree within their errors ( 0.1 mas for both optical and radio results). Radio peaks and stars are therefore clearly associated.

Overlapping the primary star in Fig. 1 to the SW feature of the radio image with highest signal-to-noise ratio (i.e. Fig. 2E) not only the position of the secondary automatically resulted to be centered on the brightest radio peak of the NE feature of that image but moreover, the position of the two stars resulted to be close to the two radio peaks in all the other images. In order to avoid a bias on the primary's position we repeated this procedure overlapping, this time, the secondary star in Fig. 1 with the NE feature of Fig. 2E. The difference between the two determinations of the stellar positions in all images was at the most 0.08 mas $\left(1.2 R_{*}\right)$. The stellar positions, given as a cross in the radio images of Fig. 2, were chosen at the center between the two determinations.

\subsection{Helmet streamers}

Whereas on the first day, (March 11, 2004, Fig. 2A), no clear signal is detected, on the second day, (March 12, 2004, Fig. 2B), two structures (NE and $\mathrm{SW}$ ) with a similar morphology are present. The NE structure is formed by two components, "a" and "b", separated by $17 R_{*}$ and the SW one is formed by two components, "c" and "d", separated by $19 R_{*}$. Both structures are therefore within the range $15 R_{*} \leq H \leq 26 R_{*}$ predicted for the size of the streamers so that interbinary coronal collisions can take place.

In all the panels of Fig. 2 it is clear that the location of the two stars is always close to the strongest peak of the NE and SW features. This coincidence indicates that the peaks "a" and "c" in Fig. 2B, are the helmets of the helmet streamers, i.e. the cusp-shaped field line located on top of a stellar sized coronal loop. Two facts indicate that Fig. 2B shows two helmet streamers just entering in collision (or immediately after): $i$ ) both structures are well distinguished and each is clearly associated with one of the stars, whereas $24 \mathrm{~h}$ earlier they were absent (Fig. 2A), and $i$ ) the structures are nearly parallel. This is the very first direct evidence revealing that even if the flare origin is magnetic reconnection due to interbinary collision, both stars emit independently in the radio range with structures of their own.

The general scenario could be the following: each of the two stars has developed solar-like helmet streamers and due to the rotation of the two stars (see Sect. 3.3) the streamers approach each other inducing a compression of the magnetic field lines and therefore, a successive change of the topology of the two magnetic configurations. Magnetic reconnection is a possible and probable consequence of this process as observed for some solar flares triggered by interactions between new and older emergences of magnetic flux in the same area (Nishio et al. 1997; Massi 2007).

The relativistic particles, produced by magnetic reconnection at the top of the streamer, spiralling around magnetic field lines emit radio synchrotron radiation (Massi et al. 2006). The particles travel along the streamer with low magnetic field strength $B_{\mathrm{S}}$, down towards the star to the helmet ("a" and "c" in Fig. 2B), with magnetic field $B_{\mathrm{H}}$, where $B_{\mathrm{H}}>B_{\mathrm{s}}$. The helmet will therefore act as a mirror point for the particles with pitch angle $\theta>\arcsin \left(B_{\mathrm{s}} / B_{\mathrm{H}}\right)^{1 / 2}$ reflecting them back into the streamer (Melrose \& Brown 1976). In the absence of any confinement, i.e. in an open streamer, the particles would leave the structure of $17-19 R_{*}$ in about $18 R_{*} / \mathrm{c} \simeq 85 \mathrm{~s}$. Since the emission is observed for a much longer time interval, at least partial confinement must exist. Postulating the presence of a second mirror, far out from the stellar surface at the top of the streamer, Massi \& collaborators (2006) modelled the observed fading of the emission after a giant flare of $360 \pm 17 \mathrm{mJy}$ as a continuous slow leakage of emitting electrons during each reflection between the two mirror points, one close $\left(\leq 5 R_{*}\right)$ to the stellar surface, i.e. the helmet, and the second far out at the top of the streamer at a distance of up to $20 R_{*}$. The two peaks "b" and "d" in Fig. 2B are the observational evidence of these postulated upper mirror points. They are in fact very strong due to the recent interaction between the two stars magnetic field and their resulting compression (see Sect. 3.4).

\subsection{Colliding helmet streamers anchored on rotating stars}

The image of March 13 (Fig. 2C) cannot be related in a clear way to the image of March 12 (Fig. 2B). The flux density of the SW structure and the total flux observed with the Effelsberg 100-m radio telescope (see Fig. 3) are higher in Fig. 2C than in Fig. 2B. This means that at the time of the earlier image either a flare had just started, and its peak was reached between March 12 and 13 or, another flare occurred within the 24 h separating the images, and it is seen in its decaying phase in the image of March 13. On March 14 (Fig. 2D) there are traces of a new upper mirror point in the NE indicating that a new flare has occurred before this observation. There are therefore 2-3 flares in 3 days, i.e. a flaring rate of up to one flare per day. How does this fit with the stellar rotation?

Rydgren \& Vrba (1983) monitored the system with UBVRI photometry and observed light variations with a period of about 3 days. Besides, using the radii of the two stars, $R_{\text {primary }}=2.22 R_{\odot}$ and $R_{\text {secondary }}=1.74 R_{\odot}($ Boden et al. 2007), 

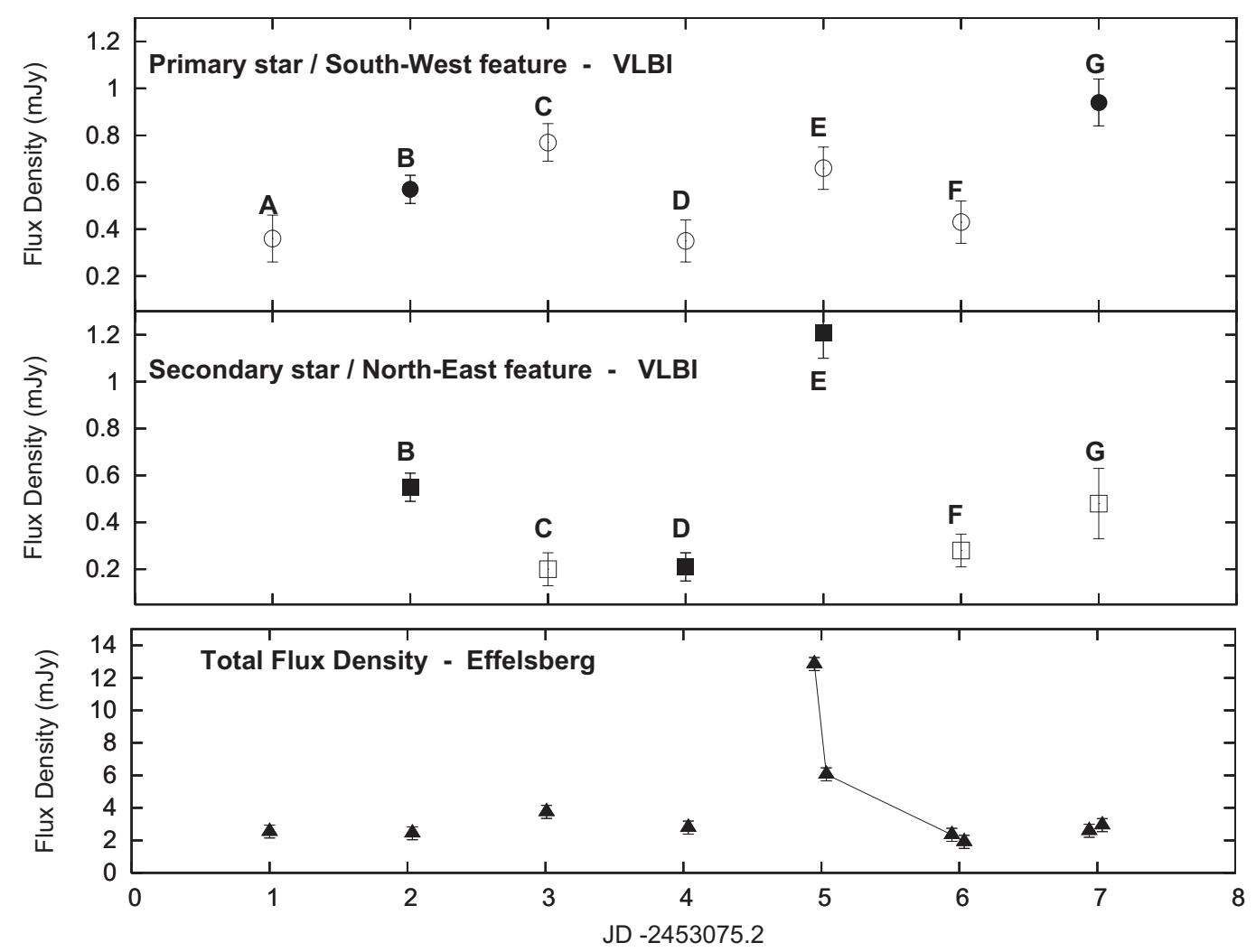

Fig. 3. Flux density versus time. Top panel: flux density of the SW radio structures (associated with the primary star) in the VLBA+EB maps. Filled circles express the combined flux of helmets and upper mirror points; empty circles indicate that only helmets are present. Middle panel: flux density of the NE radio structures (associated with the secondary star). Filled squares when helmets and upper mirrors are both present, empty squares when only helmets are present. Bottom panel: total flux density measured with the Effelsberg 100-m telescope outside the VLBA+EB observing time. The points which are likely to belong to the same flaring event have been connected (days 5-6).

their projected rotational velocities, $41.4 \mathrm{~km} \mathrm{~s}^{-1}$ and $41.9 \mathrm{~km} \mathrm{~s}^{-1}$ (Welty 1995), assuming that the spin axes of the stars are parallel to the axis of the orbit and using the orbital inclination angle $i=65.9^{\circ}$, we obtain a rotational period of 2.48 days and 1.92 days for the primary and secondary respectively.

A geometry where the spin axes of the stars are parallel to the axis of the orbit, implies interbinary collisions only if the streamers are located around the equator. This is likely to be the case, since in the Sun helmet streamers are located on the equator during the solar minimum of activity and in all the latitudes during the maximum of activity (Gibson 2001).

Therefore, following the solar analogy, it is clear that the helmet streamer configuration around each star in V773 Tau could be more complex than what is shown in the sketch of Fig. 2. This can also be inferred from the fact that a rotational period of 1.92 days for the secondary implies a rotation of about $180^{\circ}$ day. Assuming the simplest configuration of Fig. 2, this rotation would imply that an emitting helmet-upper mirror point structure should always appear in the same position angle since all the images were taken every $24 \mathrm{~h}$. Instead the position angles in Figs. $2 \mathrm{~B}, \mathrm{D}$, and $\mathrm{E}$, are $208^{\circ}, 308^{\circ}$ and, $273^{\circ}$, respectively (North throw East). A solar-like streamer belt giving more than 1-2 flares per rotation could be a straightforward explanation. Only a new set of observations lasting longer than 5.5. h each will lead to a better understanding of this complex topology.

\subsection{Short-living upper mirror points}

It is worth noting that the flux density in the VLBA+EB images never reaches even $50 \%$ of the total flux density observed with the Effelsberg 100-m telescope with a beam of $\sim 1.5$ arcmin (see Fig. 3). Most of the flux is therefore emitted from electrons that are trapped in magnetic structures which are clearly larger than the milliarcsecond features imaged by the VLBA+EB. On March 11 we observed just one helmet and a day later two complete helmet streamers were present. Nevertheless, on both days the Effelsberg 100-m telescope measured the same total flux density of 2.4 mJy. However, whereas on March 11 only $14 \%$ of this flux is in the VLBA+EB image, on the image of March 12 there is a $46 \%$ of the total flux. This seems to indicate that the upper mirror points gradually relax on larger structures which are still confining the emitting electrons but are not "visible" anymore at the milliarcsecond scales of the VLBI.

On March 15 (Fig. 2E) one helmet and its related upper mirror point can be clearly distinguished in the NE structure but only the helmet is present in the SW one. Thanks to the monitoring at the Effelsberg 100-m telescope we know that at the time of this image the flare is already in its decaying phase. In fact, while a total flux density of $12 \mathrm{mJy}$ was measured with the Effelsberg 100-m telescope two hours before the VLBA+EB observation, only 6 mJy were measured at the beginning of this observation (Fig. 3). It is significant that in Fig. 2B, that is just after/during a collision, the sources are point-like, whereas in Fig. 2E, showing an already evolved situation, the sources are extended. Relativistic electrons which diffuse from the relaxing upper mirror points remain trapped in progressively larger structures, intermediate between the VLBA+EB milliarcsecond scale and the Effelsberg 100-m telescope arcminute scale. It is worth to note that in the flare decay scenario of Fig. 2E the NE elongated structure on the secondary star is not parallel to the 
helmet streamers seen in the B run but clearly rotated. There seems to be three facts indicating that time has elapsed from the streamer-streamer collision: the clear rotation of the streamers with respect to the line joining the two stars, their non point-like appearence, and the eventual absence of upper mirror point(s). With this respect, in the last observation of March 17 (Fig. 2G), the facts that on one hand the helmet streamer anchored on the primary star appears substantially rotated and extended and on the other hand, there is a lack of the upper mirror in the NE structure, suggest a flare decay scenario as described for Fig. 2E.

The fact that after a certain time interval from the occurrence of a flare only the helmet tend to remain implies that its corresponding upper mirror point either already relaxed to a larger structure or that an upper mirror point might not even have been created. In both cases, the result is that the upper mirror point is clearly not a permanent feature.

What could be the origin of these short-living upper mirror points at distances of tens of stellar radii? Up to now there is no known solar analogy to these upper mirror points in the solar streamers. On other hand, it is known that the solar rotation pulls the magnetic field lines into a spiral shape (Parker 1958, 1969) and a much stronger effect could be expected in V773 Tau due to the faster rotation of its stellar components (more than one order of magnitude higher than the solar rotation). The faster rotation could lead to strongly curved streamers anchored on the same star to interact with each other. The feasibility of such an interaction can be verified by computing the Alfvén radius, that is the stellar distance where the magnetic field lines become to be curved by star rotation (André 1988):

$$
\frac{R_{\mathrm{A}}}{R_{*}}=26\left(\frac{B_{*}}{10^{4} \mathrm{G}}\right)^{\frac{1}{3}}\left(\frac{\dot{M}}{10^{-10} M_{\odot} \mathrm{yr}^{-1}}\right)^{-\frac{1}{6}}\left(\frac{v_{\infty}}{10^{3} \mathrm{~km} \mathrm{~s}^{-1}}\right)^{\frac{1}{6}}\left(\frac{P}{1 \mathrm{~d}}\right)^{\frac{1}{3}}
$$

The result is that for a mass loss of $10^{-13} M_{\odot} \mathrm{yr}^{-1}$ (Guenther \& Emerson 1997), a terminal velocity of a few hundred kilometers per second, $P \simeq 2 \mathrm{~d}$, and a magnetic field strength at the stellar surface of $2600 \mathrm{G}$ (Bower et al. 2003), the Alfvén radius is $\sim 60 R_{*}$. This value that clearly exceed the observed upper mirror points position rules out this self-interaction as a possible explanation and leaves as the only alternative the fact that interbinary collision produces the upper mirror points.

\section{Conclusions}

\subsection{Halo and giant flares}

The complete extent of the helmet streamers is the observed value of $18 R_{*}$, between helmet and upper mirror point, plus the distance of the helmet itself from stellar centroid. The flare activity observed on March 17 when the orbital separation was $48 R_{*}$ implies a complete extent of $\sim 24 R_{*}$ and therefore helmets located on top of coronal loops of $\sim 5 R_{*}$ as expected on the basis of the theoretical predictions (see Sects. 1 and 3.2). This explains the practically permanent flaring activity in V773 Tau A, from a level of a few mJy around apoastron $\left(52 R_{*}\right)$ to more than $100 \mathrm{mJy}$ at periastron (30 $R_{*}$ ) (Massi et al. 2002). At the short periastron distance the two coronae of $\sim 24 R_{*}$ overlap, giving rise to the observed giant flares (i.e. up to $360 \mathrm{mJy}$ at $\lambda 3 \mathrm{~mm}$, Massi et al. 2004). The result of such a situation, where the whole streamer participates in the magnetic reconnection process, is a large structure, which holds the emission peaks at the position of the two helmets anchored on each of the two stars as in the VLBI image of Phillips \& collaborators (1996) discussed in Sect. 1.

\subsection{Summary}

To what extent can our knowledge of the Sun guide us to understand other stars? Here we have reported the discovery of solar-like helmet streamers anchored on the two stars of a very young binary system, V773 Tau A. The extended helmet streamers, observed at radio wavelengths with very high resolution, cover the interbinary distance. During the stellar rotation these helmet streamers come into collision producing periodical flares. Electrons, relativistically accelerated in the collision, remain trapped in the helmet streamers and emit synchrotron radiation observed in the radio band.

The enigmatic magnetic semi-open structures typical of helmet streamers, until now observed only on the Sun, have been postulated to play a key role not only in the processes of coronal mass ejection and slow solar wind (Wiegelmann et al. 2000; Schwenn 2006), formation of prominences at large height in rapidly rotating stars (Jardine \& van Ballegooijen 2005), but also in the processes of jet formation in microquasars/AGN (de Gouveia dal Pino 2005) and outflows in young stellar objects (Ostriker \& Shu 1995; Ferreira et al. 2006). Their discovery in stars other than the Sun, at a wavelength observable at high resolution, will therefore lead to a deeper understanding of these important physical processes.

Acknowledgements. Based on observations with the Effelsberg 100-m telescope of the Max-Planck-Institut für Radioastronomie (MPIfR) and the Very Long Baseline Array (VLBA). The VLBA is a facility of the National Radio Astronomy Observatory (NRAO), operated by Associated Universities Inc. under a cooperative agreement with the National Science Foundation (NSF). We thank Peter L. Biermann, Nicolas Grosso, Giannina Poletto, and Thomas Preibisch for interesting suggestions. We are grateful to the referee Manuel Güdel for suggestions and comments which helped clarifying our manuscript on several important points.

\section{References}

André, P., Montmerle, T., Feigelson, E. D., Stine, P. C., \& Klein, K. 1988, ApJ, 335,940

Boden, A. F., Torres, G., Sargent, A. I., et al. 2007, ApJ, 670(2), in press [arXiv: 0706.2376]

Bower, G. C., Plambeck, R. L., Bolatto, A., et al. 2003, ApJ, 598, 1140

de Gouveia dal Pino, E. M. 2005, Magnetic Fields in the Universe: From Laboratory and Stars to Primordial Structures, AIPC, 784, 183

Feigelson, E. D., Welty, A. D., Imhoff, C., et al. 1994, ApJ, 432, 373

Ferreira, J., Dougados, C., \& Cabrit, S. 2006, A\&A, 453, 785

Gibson, S. E. 2001, Space Sci. Rev., 97, 69

Guenther, E. W., \& Emerson, J. P. 1997, A\&A, 321, 803

Jardine, M., \& van Ballegooijen, A. A. 2005, MNRAS, 361, 1173

Massi, M. 2007, Mem. Soc. Astron. Ital., 78, 247

Massi, M., Menten, K., \& Neidhöfer, J. 2002, A\&A, 382, 152

Massi, M., Forbrich, J., Menten, K. M., et al. 2006, A\&A, 453, 959

Melrose, D. B., \& Brown, J. C. 1976, MNRAS, 176, 15

Nishio, M., Yaji, K., Kosugi, T., Nakajima, H., \& Sakurai, T. 1997, ApJ, 489, 976

Ostriker, Eve C., \& Shu, Frank H. 1995, ApJ, 447, 813

Parker, E. N. 1958, ApJ, 128, 664

Parker, E. N. 1969, Space Sci. Rev., 9, 325

Phillips, R. B., Lonsdale, C. J., Feigelson, E. D., \& Deeney, B. D. 1996, ApJ, 111,918

Rydgren, A. E., \& Vrba, F. J. 1983, ApJ, 267, 191

Schwenn, R. 2006, Space Sci. Rev., 124, 51

Skinner, S. L., Guedel, M., Koyama, K., \& Yamauchi, S. 1997, ApJ, 486, 886

Suess, S., \& Nerney, S. 2004, Adv. Space Res., 33, 668

Tsuboi, Y., Koyama, K., Murakami, H., et al. 1998, ApJ, 503, 894

Vourlidas, A. 2006, Solar Activity and its Magnetic Origin, ed. V. Bothmer, \&

A. Hady (Cambridge University Press), 197

Welty, A. D. 1995 , AJ, 110, 776

Wiegelmann, T., Schindler, K., \& Neukirch, T. 2000, Sol. Phys., 191, 391 